\title{
Acessibilidade e trajetórias de cuidado para crianças com internações por condições sensíveis à atenção primária
}

\author{
Accessibility and care pathways for children admitted to hospital \\ for ambulatory care sensitive conditions
}

Harley Medawar Leão (https://orcid.org/0000-0002-7960-0214) ${ }^{1}$

Antônio Prates Caldeira (https://orcid.org/0000-0002-9990-9083) ${ }^{1}$

${ }^{1}$ Programa de PósGraduação em Ciências da Saúde, Universidade Estadual de Montes Claros. Campus Universitário Professor Darcy Ribeiro s/n, Vila Mauriceia. 39401089 Montes Claros MG Brasil. antonio.caldeira@ unimontes.br

\begin{abstract}
The aim of this study was to analyze health service accessibility and the care pathways of children admitted to hospital for ambulatory care sensitive conditions (ACSCs). A cross-sectional study was conducted of a random sample of children hospitalized over a period of one year in a town in the north of Minas Gerais, Brazil. The Primary Care Assessment Tool Child Edition, adapted and validated for use in Brazil (PCAT-CE), was used to assess accessibility and interviews were conducted with the children's carers to determine the pathway taken to hospitalization. Of the 376 pediatric hospitalizations assessed, 109 (28.9\%) were classified as ACSC admissions. Health service accessibility and utilization scores were low for both the ACSC and non-ACSC groups. No statistically significant differences $(p<0.05)$ were found between the two groups. The care pathways reveal that most families visited hospitals as the first service and that visits to other health services were frequent. Well over half (63.3\%) of the families of children admitted for ACSCs did not seek primary health services at any time. Accessibility was poor among the study group, regardless of the reason that led to hospitalization. The care pathways reveal an irregular pattern of service utilization for children admitted for ACSCs, suggesting a fragile health care network.
\end{abstract}

Key words Access to health services, Primary Health Care, Children's health
Resumo Objetivou-se analisar a acessibilidade e trajetórias de cuidado para crianças com internações por condições sensiveis à atenção primária (ICSAP). Trata-se de estudo transversal de internações selecionadas ao longo de um ano, em cidade do norte de Minas Gerais. Utilizou-se o Instrumento de Avaliação da Atenção Primária (PCA-Tool Brasil, versão criança), para aferir a acessibilidade e entrevistas com os cuidadores para definição detalhada do percurso familiar até a internação. Entre as 379 hospitalizações avaliadas, 109 (28,9\%) foram classificadas como ICSAP. Os escores de acessibilidade e utilização dos serviços de saúde foram baixos tanto para as ICSAP como para as demais internações e sem diferenças estatisticamente significantes $(p<0,05)$. As trajetórias de cuidado revelam que a maioria das familias procurou primeiramente o hospital, sendo frequente a busca por vários serviços de saúde, antes da hospitalização. Em 63,3\% dos casos de ICSAP, as famílias não procuraram a atenção primária em nenhum momento. A acessibilidade se mostrou comprometida para o grupo estudado, independente do motivo que gerou a internação $e$ as trajetórias de cuidado revelaram um padrão irregular de utilização dos serviços de saúde para crianças com ICSAP, sugerindo uma rede assistencial frágil.

Palavras-chave Acesso aos serviços de saúde, Atenção Primária à Saúde, Saúde da criança 


\section{Introdução}

Nas últimas décadas, o Brasil vivenciou uma substancial melhoria nos indicadores de saúde materno-infantil ${ }^{1}$. Muitos avanços têm sido atribuídos à ampliação do acesso, com a expansão da Estratégia Saúde da Família (ESF)². Todavia, alguns desafios permanecem e salientam a complexidade dos cuidados em saúde, como a elevada taxa de prematuridade, a mortalidade neonatal, a estabilização dos indicadores de aleitamento materno e a persistência da sífilis congênita ${ }^{1}$.

$\mathrm{Na}$ atenção à saúde infantil, as hospitalizações representam um importante problema, que implica elevados custos, sofrimento familiar e que acomete desigualmente a sociedade, com frequências mais elevadas para as crianças de famílias mais pobres ${ }^{3}$. Parte das internações são decorrentes de falhas do atendimento ambulatorial, que, se atendidos adequada e oportunamente no primeiro nível de atenção, poderia evitar o agravamento da condição clínica e reduzir as hospitalizações. Essas situações definem as chamadas Internações por Condições Sensíveis à Atenção Primária (ICSAP) ${ }^{4}$.

A lista das condições sensíveis à atenção primária representa situações que podem ser bem manejadas por equipes da Atenção Primária em Saúde (APS) e cujas hospitalizações seriam indicadores de falta de acesso e/ou qualidade deficitária dos cuidados em saúde 4 . Nos últimos anos, vários estudos correlacionam as taxas de ICSAP com a ampliação das equipes da ESF ${ }^{5-7}$. Todavia, a maior parte desses estudos apresenta correlações ecológicas, que não permitem assegurar, de forma inequívoca, uma influência forte e exclusiva da atuação das equipes da ESF para redução das taxas de ICSAP. Alguns aspectos que definem o padrão de utilização dos serviços de saúde estão fora do escopo da APS, o que comprometeria o papel da ESF como determinante das ICSAP ${ }^{8,9}$.

As ICSAP na infância incluem, sobretudo, condições agudas e existem questionamentos também sobre o alcance das ações das equipes de APS em relação à evitabilidade dessas internações ${ }^{10}$. Um estudo conduzido na região Nordeste do Brasil registrou que, em um contexto de grande ampliação das equipes da ESF, a proporção de hospitalizações nos principais grupos de causas por condições sensíveis se manteve ou aumentou, ao longo da década analisada, o que reforça a interveniência de outros fatores ${ }^{11}$.

A análise da literatura registra que existem algumas lacunas sobre a relação ICSAP e ampliação da oferta de acesso pela ESF. Algumas delas podem ser potencialmente preenchidas a partir de um maior conhecimento do percurso desenvolvido pela criança e por sua família desde o adoecimento até a hospitalização, definidas como trajetórias de cuidado.

Avaliações das trajetórias de cuidado são distintas das avaliações de Itinerários Terapêuticos, que possuem abordagem eminentemente qualitativa, com análise de aspectos culturais e representações sociais sobre o processo saúde-doença. Muitas vezes, essas avaliações não consideram questões como o acesso, a utilização dos serviços e fatores relacionados ao contexto do paciente ${ }^{12}$. Estudos sobre as trajetórias de cuidado podem auxiliar para a qualificação da assistência, ao apontar falhas ao longo do percurso assistencial, considerando que as ICSAP representam desfechos indesejáveis e potencialmente evitáveis. $\mathrm{O}$ objetivo do presente estudo foi analisar a acessibilidade e as trajetórias de cuidado para crianças com ICSAP.

\section{Métodos}

Trata-se de um estudo transversal, com identificação incidental de hospitalizações infantis ao longo de um ano, em uma amostra representativa, em uma cidade do interior do Sudeste brasileiro. O município contava, em 2017, com população residente de aproximadamente $400 \mathrm{mil}$ habitantes, com cerca de 80 leitos para internação hospitalar infantil pelo Sistema Único de Saúde (SUS), distribuídos em dois hospitais e uma cobertura da ESF superior a $80 \%$.

A população do estudo foi definida por crianças hospitalizadas, com idade entre zero e dez anos completos, residentes na cidade sede do estudo, acompanhadas por pais ou responsáveis no momento da abordagem para coleta de dados. Foram excluídos os casos de crianças oriundas de outros municípios e cujos acompanhantes tinham idade menor de 18 anos.

O cálculo amostral foi realizado a partir dos seguintes parâmetros: nível de confiança de 95\%, margem de erro de $5 \%$, prevalência estimada de $40 \%$ de ICSAP, considerando, para cada hospital, o número de leitos hospitalares pediátricos e o número de internações pediátricas ocorridas no último ano. Ao valor mínimo estimado, acrescentou-se $10 \%$ para perdas e recusas. Assim, estimou-se uma amostra mínima de 375 hospitalizações.

O instrumento de coleta de dados constava de variáveis sociodemográficas (idade, sexo, cor 
da pele, escolaridade, renda e cidade de origem) e assistenciais (diagnósticos, históricos de internações, serviços e profissionais procurados e dados do prontuário clínico do paciente), além de avaliação de "acessibilidade" e "utilização dos serviços de atenção primária”. Esses itens foram retirados do Instrumento de Avaliação da Atenção Primária (PCATool-Brasil, versão Criança), validado para o português brasileiro a partir de instrumento internacional, que atribui escores aos atributos essenciais e derivados a atenção primária. Habitualmente, um escore acima de 6,6 (em uma escala de zero a dez) para cada atributo é considerado como satisfatório ${ }^{13}$. $\mathrm{O}$ atributo "acesso de primeiro contato" é um dos atributos essenciais da atenção primária e compreende as dimensões "acessibilidade" (com seis questões) e "utilização" (com três questões) que foram empregadas neste estudo.

Os diagnósticos clínicos foram coletados dos prontuários e codificados segundo a décima revisão da Classificação Internacional de Doenças e Causas de Morte (CID-10). Em seguida, foram classificados pelo grupo, conforme a lista brasileira de condições sensíveis à atenção primária ${ }^{6}$ em ICSAP e não-ICSAP. A lista das ICSAP foi definida após pesquisa que contou em seu processo de validação, com a participação de profissionais de saúde, gestores, pesquisadores e consulta pública, definindo, ao final uma relação de vinte grupos diagnósticos ${ }^{4}$.

A identificação das crianças hospitalizadas foi realizada por amostragem aleatória simples, com intervalos médios de sete dias para as buscas nos hospitais. A equipe de coleta, formada por um enfermeiro e dois estudantes de enfermagem, foi treinada e, a cada visita hospitalar, todas as crianças internadas que atendiam aos critérios de inclusão eram selecionadas para o estudo. A aplicação dos instrumentos foi realizada entre junho de 2016 e agosto de 2017. O período de coleta foi estimado com o objetivo de se evitar o viés de seleção, evitando a influência das variações sazonais das doenças. Para fins de controle de qualidade, foram realizadas 20 (vinte) reentrevistas, aleatoriamente selecionadas, verificando-se a fidelidade das informações.

Os dados foram analisados por meio do programa estatístico IBM SPSS (Statistical Package for the Social Sciences) versão 22.0 for Windows. As proporções das características em cada grupo (ICSAP e não-ICSAP) foram comparadas por meio do teste qui-quadrado de Pearson. Foram realizadas comparações das médias dos escores de acessibilidade e demais médias por meio do teste U de Mann-Whitney. A normalidade dos dados foi aferida pelo teste de Kolmogorov-Smirnov. O nível de significância assumido foi de $5 \%(\mathrm{p}<0,05)$.

Para as crianças hospitalizadas por condições sensíveis, foi realizada uma entrevista detalhada com o cuidador responsável, identificando todos os pontos de atenção à saúde utilizados e o intervalo desde os primeiros sintomas até a internação. $\mathrm{O}$ consolidado dessas entrevistas permitiu a elaboração do fluxograma das trajetórias de cuidado para as crianças com ICSAP. Na avaliação das trajetórias de cuidado, analisou-se o referenciamento (encaminhamento) pela ESF e procura por livre demanda pelo serviço hospitalar para as crianças hospitalizadas.

O projeto de pesquisa foi aprovado pelo Comitê de Ética em Pesquisa da Universidade Estadual de Montes Claros e pelos Centros de Ensino e Pesquisa dos hospitais envolvidos. Todos os participantes foram entrevistados apenas após a assinatura do Termo de Consentimento Livre e Esclarecido.

\section{Resultados}

Foram avaliadas 376 hospitalizações de crianças, $109(28,9 \%)$ classificadas como ICSAP, tendo como principais causas: "pneumonia bacteriana” (45,9\%), "infecção no rim e trato urinário" $(14,7 \%)$, "gastroenterites infecciosas e complicações” (11,0\%) e bronquite/asma (11,0\%).

Tanto no grupo de crianças com ICSAP como no grupo das demais condições, o principal cuidador informado foi a mãe, a idade materna predominante foi de 20 a 39 anos e os pais residiam na mesma casa. Registrou-se baixo percentual de pais e mães com escolaridade igual ou superior a 12 anos e mais de dois terços das famílias em ambos os grupos referiam renda familiar inferior a dois salários mínimos. Os grupos se mostraram estatisticamente diferentes apenas em relação ao percentual de crianças do sexo masculino, que foi maior para as hospitalizações não-ICSAP $(\mathrm{p}=0,003)$ (Tabela 1).

A média de tempo (em dias) decorridos entre o início dos sintomas até a primeira procura por serviços de saúde, bem como o tempo entre o início dos sintomas até a internação, não apresentaram diferenças significativas entre os grupos ICSAP ou não-ICSAP. Os escores de acessibilidade e de utilização, segundo o PCATool, se mostraram abaixo de 6,6 e não apresentaram diferenças estatisticamente significantes entre crianças hospi- 


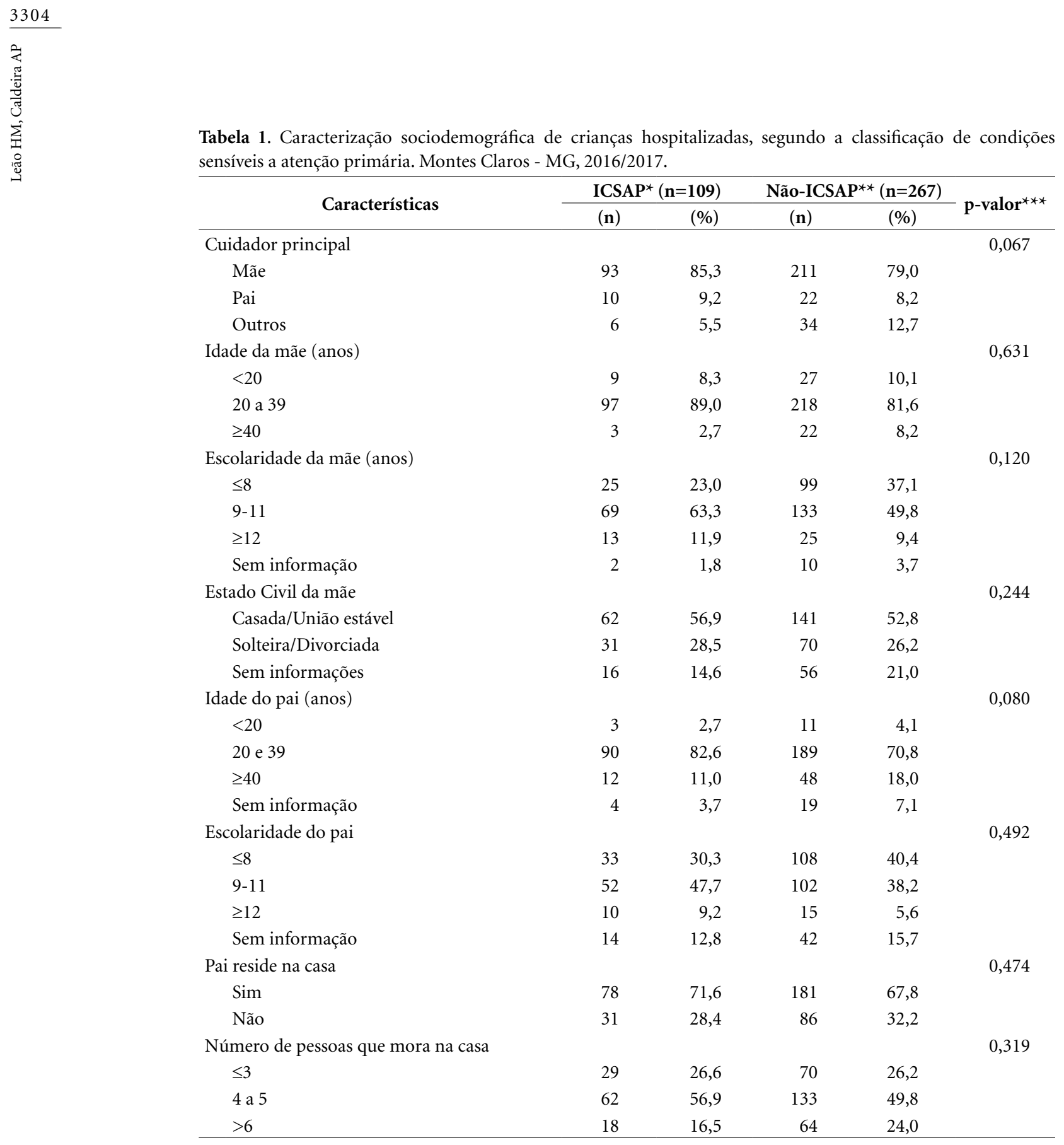

continua

talizadas por condições sensíveis ou por outras causas (Tabela 2).

A avaliação individual dos itens de acessibilidade e de utilização do PCA-Tool (Tabela 3), demonstrou que apenas a variável dificuldade de conseguir atendimento médico apresentou diferença estatisticamente significante $(p=0,045)$ entre ICSAP e não-ICSAP. Nesse caso, o maior percentual de respostas apontando dificuldades para conseguir atendimento médico foi referido pelo grupo não-ICSAP.
$\mathrm{Na}$ análise das trajetórias de cuidado, observou-se que as crianças que foram internadas e buscaram a ESF como último serviço antes da internação, foram encaminhadas para hospitalização em $53,8 \%$ para as ICSAP e $56,5 \%$ para as não-ICSAP.

A Figura 1 mostra um fluxograma com a síntese das trajetórias referidas pelas famílias em relação à busca por cuidados de saúde, apenas para os casos de ICSAP. É possível verificar que a maioria das famílias procurou a assistência hos- 
Tabela 1. Caracterização sociodemográfica de crianças hospitalizadas, segundo a classificação de condições sensíveis a atenção primária. Montes Claros - MG, 2016/2017.

\begin{tabular}{|c|c|c|c|c|c|}
\hline \multirow{2}{*}{ Características } & \multicolumn{2}{|c|}{$\operatorname{ICSAP}^{\star}(n=109)$} & \multicolumn{2}{|c|}{ Não-ICSAP ${ }^{\star *}(n=267)$} & \multirow{2}{*}{ p-valor $* * *$} \\
\hline & $(\mathbf{n})$ & $(\%)$ & $(\mathbf{n})$ & $(\%)$ & \\
\hline Renda Familiar (em salários mínimos) $\dagger$ & & & & & 0,779 \\
\hline$\leq 1$ & 48 & 44,0 & 128 & 47,9 & \\
\hline 1,1 a 2 & 37 & 33,9 & 82 & 30,7 & \\
\hline$>2,0$ & 15 & 13,7 & 31 & 11,6 & \\
\hline Sem informação & 9 & 8,4 & 26 & 9,7 & \\
\hline Sexo da criança & & & & & 0,003 \\
\hline Masculino & 41 & 37,6 & 145 & 54,3 & \\
\hline Feminino & 68 & 62,4 & 122 & 45,7 & \\
\hline Idade da criança (anos) & & & & & 0,744 \\
\hline$\leq 1$ & 40 & 36,7 & 110 & 41,2 & \\
\hline 1,1 a 3 & 31 & 28,4 & 43 & 16,1 & \\
\hline$>3,1$ & 38 & 34,9 & 114 & 42,7 & \\
\hline Cor da Pele da criança & & & & & 0,210 \\
\hline Branca & 28 & 25,7 & 51 & 19,1 & \\
\hline Pardo/Preta & 76 & 69,7 & 203 & 76,0 & \\
\hline Amarela/Indígena & 5 & 4,6 & 13 & 4,9 & \\
\hline
\end{tabular}

Tabela 2. Comparação entre períodos decorridos para a atendimentos e entre escores PCATool de crianças hospitalizadas, segundo a classificação de condições sensíveis a atenção primária. Montes Claros - MG, 2016/2017.

\begin{tabular}{lrrrrr}
\multicolumn{1}{c}{ Características } & \multicolumn{2}{c}{$\begin{array}{l}\text { ICSAP } \\
\text { (n=109) }\end{array}$} & \multicolumn{2}{c}{$\begin{array}{c}\text { Não-ICSAP } \\
\text { (n=267) }\end{array}$} & p-valor $^{* * *}$ \\
\cline { 2 - 5 } & Média & \multicolumn{1}{c}{ DP } & Média & DP & \\
\hline Tempo decorrido para atendimento (em dias) & & & & & \\
Entre início dos sintomas e primeiro atendimento & 0,85 & 1,46 & 2,22 & 8,30 & 0,975 \\
Entre início dos sintomas e hospitalização & 6,99 & 12,72 & 10,80 & 26,18 & 0,772 \\
Escores do PCATool & & & & & \\
Acessibilidade & 4,65 & 1,81 & 4,81 & 1,86 & 0,362 \\
Utilização & 6,57 & 2,48 & 6,49 & 2,84 & 0,391
\end{tabular}

*ICSAP: Internações por Condições Sensíveis à Atenção Primária; ${ }^{* * N a ̃ o-I C S A P: ~ I n t e r n a c ̧ o ̃ e s ~ p o r ~ C o n d i c ̧ o ̃ e s ~ N a ̃ o ~ S e n s i ́ v e i s ~ a ̀ ~}$ Atenção Primária; ${ }^{* * * T e s t e ~ U ~ d e ~ M a n n-W h i t n e y . ~}$

Fonte: Elaborado pelos autores.

pitalar como primeiro serviço $(56,0 \%)$ e cerca de um terço das famílias $(32,1 \%)$ procurou primeiramente a unidade básica de saúde. É frequente a busca por vários serviços antes da hospitalização das crianças. A ESF foi procurada como serviço assistencial para atenção à saúde da criança doente em algum momento durante a trajetória de cuidado à saúde até a hospitalização em 36,7\% dos casos de ICSAP.

\section{Discussão}

O presente estudo registrou um elevado percentual de ICSAP entre as crianças hospitalizadas, o que retrata um possível comprometimento da atenção à saúde infantil no âmbito da APS para a região estudada. Apesar do valor observado ser menor do que o registrado para a mesma região, há alguns anos, em estudo com mesma metodologia, a ampliação da cobertura de equipes de saúde da família ensejariam valores mais baixos ${ }^{10}$. 


\begin{tabular}{|c|c|c|c|c|c|c|}
\hline \multirow[t]{23}{*}{ 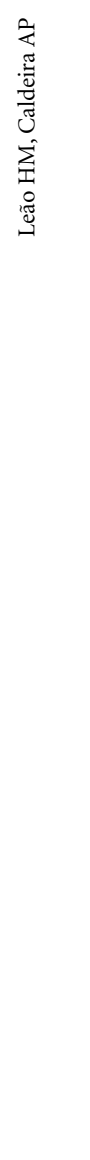 } & \multicolumn{6}{|c|}{$\begin{array}{l}\text { Tabela 3. Avaliação dos cuidadores de crianças internadas para os atributos de acesso ao primeiro contato } \\
\text { (utilização e acessibilidade) em serviços de atenção primária. Montes Claros-MG, 2016/2017. }\end{array}$} \\
\hline & \multirow{2}{*}{\multicolumn{2}{|c|}{ Acesso ao Primeiro Contato }} & \multicolumn{2}{|c|}{ ICSAP } & \multirow{2}{*}{ Total } & \multirow{2}{*}{$\begin{array}{c}\text { p- } \\
\text { valor }\end{array}$} \\
\hline & & & Sim & Não & & \\
\hline & \multicolumn{6}{|l|}{ Utilização } \\
\hline & \multirow{2}{*}{$\begin{array}{l}\text { Quando sua criança necessita de uma consulta de revisão ("consulta } \\
\text { de rotina"), você vai ao seu PSF antes de ir a outro serviço de saúde? }\end{array}$} & Sim & 87 & 204 & 291 & 0,473 \\
\hline & & Não & 22 & 63 & 85 & \\
\hline & \multirow{2}{*}{$\begin{array}{l}\text { Quando sua criança tem um novo problema de saúde, você vai ao } \\
\text { seu PSF antes de ir a outro serviço de saúde? }\end{array}$} & $\operatorname{Sim}$ & 58 & 158 & 216 & 0,288 \\
\hline & & Não & 51 & 109 & 160 & \\
\hline & \multirow{2}{*}{$\begin{array}{l}\text { Quando sua criança tem que consultar um médico especialista, o } \\
\text { seu PSF tem que encaminhá-la obrigatoriamente? }\end{array}$} & $\operatorname{Sim}$ & 74 & 176 & 250 & 0,713 \\
\hline & & Não & 35 & 91 & 126 & \\
\hline & \multicolumn{6}{|l|}{ Acessibilidade } \\
\hline & \multirow{2}{*}{$\begin{array}{l}\text { Quando o PSF está aberto e sua criança fica doente, alguém deste } \\
\text { serviço de saúde a atende no mesmo dia? }\end{array}$} & $\operatorname{Sim}$ & 57 & 151 & 208 & 0,451 \\
\hline & & Não & 52 & 116 & 168 & \\
\hline & \multirow{2}{*}{$\begin{array}{l}\text { Você tem que esperar muito tempo ou falar com muitas pessoas } \\
\text { para marcar hora no PSF? }\end{array}$} & Sim & 54 & 110 & 164 & 0,139 \\
\hline & & Não & 55 & 157 & 212 & \\
\hline & \multirow{2}{*}{$\begin{array}{l}\text { É fácil marcar hora para uma consulta de revisão da criança } \\
\text { ("consulta de rotina") no PSF? }\end{array}$} & Sim & 65 & 152 & 217 & 0,630 \\
\hline & & Não & 44 & 115 & 159 & \\
\hline & \multirow{2}{*}{$\begin{array}{l}\text { Quando você chega no PSF, tem que esperar mais de } 30 \text { minutos } \\
\text { para que sua criança consulte com o médico/ enfermeiro? }\end{array}$} & $\operatorname{Sim}$ & 79 & 174 & 253 & 0,171 \\
\hline & & Não & 30 & 93 & 123 & \\
\hline & \multirow{2}{*}{$\begin{array}{l}\text { É difícil para você conseguir atendimento médico para sua criança } \\
\text { no PSF quando você pensa que é necessário? }\end{array}$} & Sim & 61 & 119 & 180 & 0,045 \\
\hline & & Não & 48 & 148 & 196 & \\
\hline & \multirow{2}{*}{$\begin{array}{l}\text { Quando o PSF está aberto, você consegue aconselhamento rápido } \\
\text { pelo telefone se precisar? }\end{array}$} & Sim & 20 & 54 & 74 & 0,678 \\
\hline & & Não & 89 & 213 & 302 & \\
\hline
\end{tabular}

Fonte: Elaborado pelos autores.

Alguns estudos apontam uma diminuição das ICSAP nos últimos anos no Brasil, relacionando os resultados com a expansão da ESF, numa perspectiva de associação ecológica ${ }^{5-7}$.

É razoável assumir que a ampliação do acesso disponibiliza à população em geral cuidados mais oportunos com potencial impacto sobre os indicadores de saúde, incluindo-se as internações hospitalares. Alguns autores destacam que, além da ampliação do acesso, o modelo assistencial da ESF implica mudanças da prática clínica que propiciariam cuidados mais efetivos, especialmente para pacientes com condições crônicas ${ }^{14}$. Entretanto, outros estudos destacam que a utilização das ICSAP, como indicador de resolutividade das equipes da APS, deve ser vista com cautela, considerando a não observância de aspectos socioeconômicos, relacionados à gravidade do quadro ou às particularidades do processo de admissão hospitalar ${ }^{15-18}$.

Efetivamente, os fatores associados às ICSAP são complexos e podem incluir aspectos próprios da população (maior privação), da prática médica (falta de continuidade dos cuidados) e da assistência hospitalar (aumento da disponibilidade de leito no hospital). Há que se considerar ainda que as taxas de ICSAP podem variar devido ao aumento da frequência de internações muito curtas, referências inadequadas, opções limitadas de cuidados, disponibilidade de leitos ou metas governamentais, tornando as ICSAP "sensíveis à oferta", 19 .

Neste estudo, as duas dimensões que avaliam $\mathrm{o}$ acesso de primeiro contato apresentaram escores considerados insatisfatórios ${ }^{13}$. Não se registrou associação entre os escores observados com a classificação das hospitalizações em ICSAP ou não-ICSAP. A necessidade de melhoria dos serviços, instiga o questionamento de outras relações de associação ou limitações do indicador ICSAP para a faixa etária. Como não foram observadas diferenças entre os escores de acesso e de utilização para os dois tipos de internações, não é possível afirmar que essas variáveis estejam definindo efetividade das equipes da ESF na evitabilidade das hospitalizações.

Quando se comparou os percentuais de cada uma das respostas do PCA-Tool, considerando o tipo de internação, a dificuldade de acesso ao atendimento médico demonstrou associação as ICSAP. Essa dificuldade da família na busca de atendimento médico na ESF pode justificar a busca por outros serviços de saúde, descaracte- 


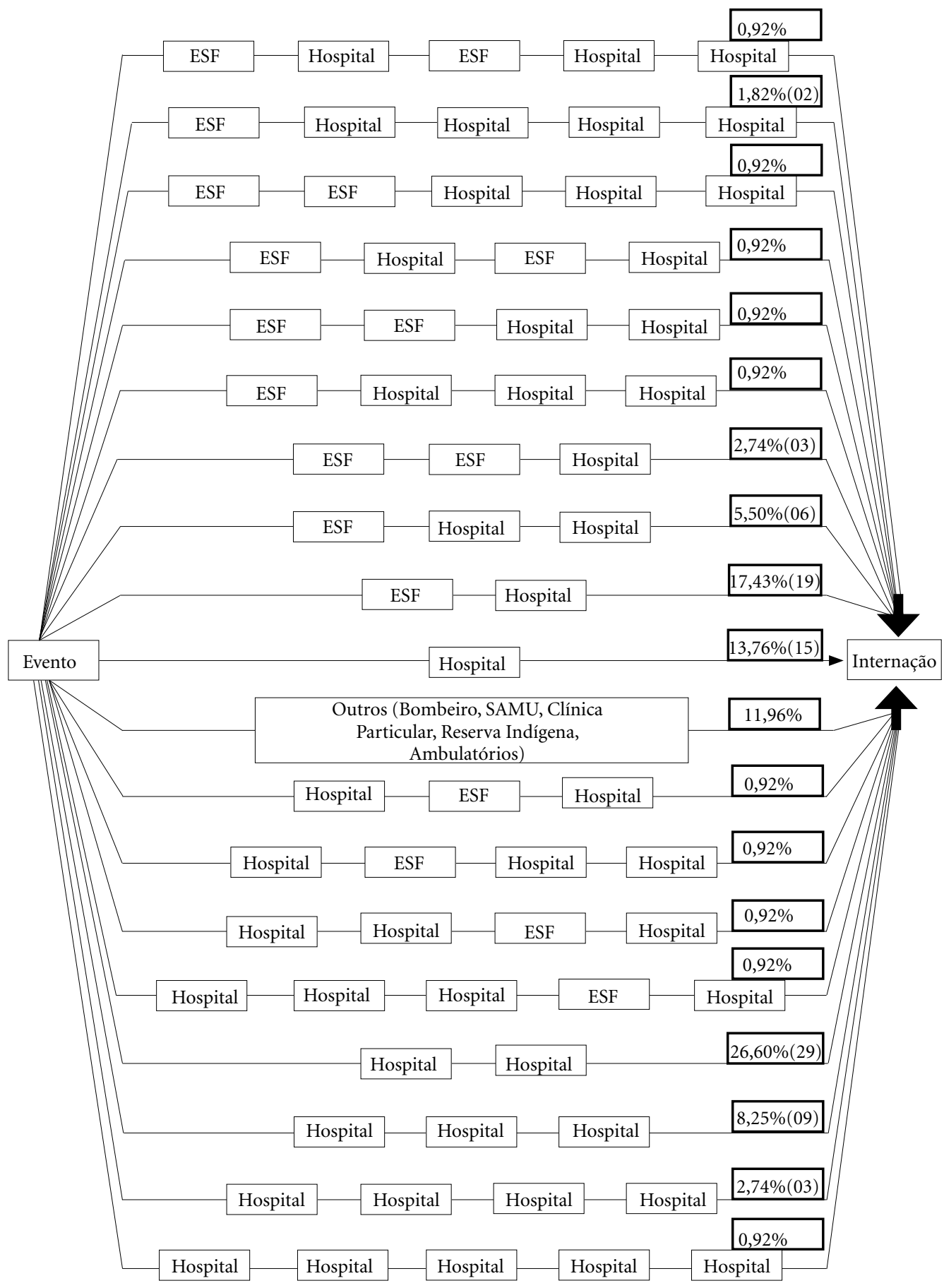

Figura 1. Fluxograma das trajetórias de cuidado referidas pelas famílias de crianças hospitalizadas por Condições Sensíveis à Atenção Primária. Montes Claros-MG, 2017.

Fonte: Elaborado pelos autores.

rizando a organização da rede e sobrecarregando os serviços hospitalares. A dificuldade em obter atendimento ocasiona o deslocamento da população para outros serviços, principalmente 
hospitalares ${ }^{20,21}$. Uma vez iniciada a atenção em hospitais e pronto-socorros, serviços que não se caracterizam como atenção primária, dificilmente estará assegurada a continuidade do cuidado. De forma consequente, haverá prejuízo do vínculo e da integralidade com ausência de responsabilização e falta de continuidade.

Para uma atenção primária forte e resolutiva é necessário um acesso facilitado, em que a pessoa vinculada àquela unidade de saúde consiga um atendimento quando precisar, no horário compatível com a disponibilidade da equipe e do paciente. Outros trabalhos destacaram fragilidades no acesso aos serviços de saúde para crianças, ofertado pelas equipes da ESF, e problemas de qualidade e de valorização dos serviços de urgên cia $^{22,23}$.

Em estudo realizado no Sul do Brasil, os autores verificaram que uma melhor qualidade dos serviços de saúde (com maiores escores do PCA-Tool), em um ambiente de serviços predominantemente de baixa qualidade e não adaptados ao atendimento das condições crônicas, não influenciou na taxa de ICSAP ${ }^{24}$. Em outro estudo de revisão sistemática, observou-se que a associação de menores taxas de ICSAP com o aumento da cobertura da ESF não é um evento claro e tem demostrado controvérsias, podendo sugerir uma superestimação do efeito da cobertura da ESF no país, seja por reduzida capacidade técnica seja por restrição de recursos humanos ${ }^{25}$.

No presente estudo, não foram observadas diferenças significativas no tempo médio, em dias, desde o início do quadro até o primeiro contato com o serviço de saúde ou até a hospitalização entre os dois tipos de internações, destaca que as condições que cursam com hospitalizações evitáveis não estão sendo reconhecidas e oportunamente cuidadas, o que foi apontado em outro estudo $^{15}$. Esse resultado ressalta a importância de se conhecer melhor a trajetória da família desde os primeiros sintomas até a internação.

A análise das trajetórias de cuidado das ICSAP permite observar que existe uma situação contrária ao modelo proposto de rede assistencial de atenção à saúde, com baixa procura pelas equipes da atenção primária em qualquer situação de agravo à saúde. Um estudo realizado nos Estados Unidos encontrou dados similares, avaliando as consultas ambulatoriais antes da internação de crianças: quase um terço das crianças não teve nenhuma consulta ambulatorial nos sete dias que antecederam a ICSAP ${ }^{26}$. É bastante relevante o fato de que a maioria das famílias com crianças com ICSAP não procurou assistência da ESF, em nenhum momento, corroborando o fato de que o acesso ao serviço hospitalar não ocorre a partir da atenção primária como coordenadora do fluxo de atenção à saúde ${ }^{27}$.

A baixa proporção de procura pelas equipes ESF pode estar relacionada a dificuldades decorrentes de horários de funcionamento, adscrição ao local de moradia ou relacionadas ao processo de trabalho das equipes para garantir simultaneamente atendimento à demanda espontânea e aos grupos não prioritários. Mas também pode ser escolha dos familiares na busca por outros serviços como uma porta de entrada para o sistema, considerando experiências passadas ou expectativas de maior resolutividade ${ }^{28}$.

As trajetórias identificadas neste estudo denotam que não parece haver reconhecimento das famílias da organização do sistema de saúde, demonstrando não existir vínculo nem coordenação do cuidado pela APS. A continuidade e a integralidade da assistência parecem ser atributos pouco reconhecidos pelas famílias no âmbito das equipes da ESF, aspectos registrados em estudo anterior, que destacava a necessidade de maior aderência aos atributos da APS por parte das equipes da $\mathrm{ESF}^{29}$.

A análise das trajetórias de cuidado por parte das famílias traz ainda a necessidade de se discutir sobre a qualidade da assistência dos serviços de atenção primária. Serviços com qualidade inferior à ideal parecem não ter impacto sobre as ICSAP ${ }^{28}$. Quando não se esgotam os recursos disponíveis ao paciente no primeiro nível de atenção antes do encaminhamento para níveis mais complexos há uma queima de etapas no processo de cuidado em saúde, apontando o fracasso dos fluxos existentes $^{29}$. A responsabilização do profissional com o sujeito atendido, nesses casos, com o estabelecimento de maior compromisso, resultaria em medidas mais adequadas às necessidades do paciente.

Outro aspecto a ser questionado é a oferta, aparentemente excedente, na atenção hospitalar, direcionando menos esforços para a gestão da atenção primária, o que, em certa medida, contribui para o agravo de doenças que poderiam ser tratadas precocemente, já que não há continuidade do cuidado, ou por internações precoces como alternativa de tratamento. Todavia, essas questões não foram objeto do presente estudo e demandam novas investigações.

As ICSAP na infância têm determinantes complexos que incluem aspectos relacionados à população assistida, aos cuidados recebidos no primeiro nível de atenção e às práticas de hospitalizações $^{18}$. Os resultados alcançados no presente estudo registram que o indicador ICSAP 
na infância retrata, sobretudo, a (des)organização da rede de saúde em prover continuidade da assistência e cuidado de qualidade à população infantil.

Algumas limitações devem ser lembradas para a interpretação e para a generalização dos dados. Trata-se de uma amostra que, embora aleatória e representativa de hospitalizações realizadas ao longo de um ano, é restrita a um único município. O fato de as análises utilizarem apenas as hospitalizações pelo SUS também representa uma limitação do estudo. Outra particularidade é que, durante o período de coleta, os hospitais envolvidos passaram por reformas em suas unidades pediátricas, gerando internações curtas alocadas nos respectivos pronto-socorros e não consideradas na análise. Todavia, os resultados são relevantes e congruentes com a literatura, denotando a necessidade de um olhar diferenciado às ICSAP na infância.

Conclui-se que a acessibilidade ainda carece de melhoras para o grupo estudado, independente do motivo que gerou a internação. Adicionalmente, existe um padrão irregular de utilização dos serviços de saúde por parte das famílias de crianças com ICSAP e o indicador não retrata a continuidade da assistência e cuidado de qualidade à população infantil.

\section{Colaboradores}

Todos os autores contribuíram igualmente para este estudo.

\section{Referências}

1. Leal MC, Szwarcwald CL, Almeida PVB, Aquino EML, Barreto ML, Barros FC, Victora CG. Saúde reprodutiva, materna, neonatal e infantil nos 30 anos do Sistema Único de Saúde (SUS). Cien Saude Colet 2018; 23(6):1915-1928.

2. Aquino R, Oliveira NF, Barreto ML. Impact of the Family Health Program on infant mortality in Brazilian municipalities. Am J Public Health 2009; 99(1):87-93.

3. Matijasevich A, Cesar JA, Santos IS, Barros AJD, Dode MASO, Barros FC, Victora CG. Internações hospitalares durante a infância em três estudos de base populacional no Sul do Brasil: tendências e diferenciais. Cad Saude Publica 2008; 24 (Supl. 3):S437-S443.

4. Alfradique ME, Bonolo PF, Dourado I, Lima-Costa MF, Macinko J, Mendonça CS, Oliveira VB, Sampaio LFR, Simoni C, Turci MA. Internações por condições sensíveis à atenção primária: a construção da lista brasileira como ferramenta para medir o desempenho do sistema de saúde (Projeto ICSAP — Brasil). Cad Saude Publica 2009; 25(6):1337-1349.

5. Pinto Júnior EP, Aquino R, Medina MG, Silva MGC. Effect of the Family Health Stategy on hospitalizations for primary care sensitive conditions in infants in Bahia State, Brazil. Cad Saude Publica 2018; 34(2):e00133816. 
6. Carvalho SC, Mota E, Dourado I, Aquino R, Teles C, Medina MG. Hospitalizations of children due to primary health care sensitive conditions in Pernambuco State, Northeast Brazil. Cad Saude Publica 2015; 31(4):744-754.

7. Pinto LF, Giovanella L. Do Programa à Estratégia Saúde da Família: expansão do acesso e redução das internações por condições sensíveis à atenção básica (ICSAB). Cien Saude Colet 2018; 23(6):1903-1913.

8. Pazó RG, Frauches DO, Molina MCB, Cade NV. Modelagem hierárquica de determinantes associados a internações por condições sensíveis à atenção primária no Espírito Santo, Brasil. Cad Saude Publica 2014; 30(9):1891-1902.

9. Botelho JF. Portela MC. Risco de interpretação falaciosa das internações por condições sensíveis à atenção primária em contextos locais, Itaboraí, Rio de Janeiro, Brasil, 2006-2011. Cad Saude Publica 2017; 33(3):e00050915.

10. Caldeira AP, Fernandes VBL, Fonseca WP, Faria AA. Internações pediátricas por condições sensíveis à atenção primária em Montes Claros, Minas Gerais, Brasil. Ver Bras Saude Mater Infant 2011; 11(1):61-71.

11. Barreto JOM, Nery IS, Costa MSC. Estratégia Saúde da Família e internações hospitalares em menores de 5 anos no Piauí, Brasil. Cad Saude Publica 2012; 28(3):515-526.

12. Cabral ALLV, Martinez-Hemáez A, Andrade EIG, Cherchiglia ML. Itinerários terapêuticos: o estado da arte da produção científica no Brasil. Cien Saude Colet 2011; 16(11):4433-4442.

13. Brasil. Ministério da Saúde (MS). Secretaria de Atenção em Saúde. Departamento de Atenção Básica. Manual do instrumento de avaliação da atenção primária à saúde: Primary Care Assessment Tool - Pcatool - Brasil. Brasília: MS, 2010.

14. Macinko J, Dourado I, Aquino R, Bonolo PDF, LimaCosta MF, Medina MG, Mota E, Oliveira VB, Turci MA. Major expansion of primary care in Brazil linked to decline in unnecessary hospitalization. Health Aff (Millwood) 2010; 29(12):2149-2160.

15. Santos VCF, Ruiz ENF, Roese A, Kalsing A, Gerhardt TE. Internações por condições sensíveis a atenção primária (ICSAP): discutindo limites à utilização deste indicador na avaliação da Atenção Básica em Saúde. RECIIS 2013; 7(2):1-16.

16. Melo MD, Egry EY. Social determinants of hospitalizations for ambulatory care sensitive conditions in Guarulhos, São Paulo. Rev Esc Enferm USP 2014; 48(esp.):129-136.

17. Nedel FB, Facchini LA, Bastos JL, Martín-Mateo M. Conceptual and methodological aspects in the study of hospitalizations for ambulatory care sensitive conditions. Cien Saude Colet 2011; 16(1):1145-1154.

18. Busby J, Purdy S, Hollingworth W. How do population, general practice and hospital factors influence ambulatory care sensitive admissions: a cross sectional study. BMC Family Practice 2017; 18(1):67.

19. Lima SAV, Silva MRF, Carvalho EMF, Cesse EAPC, Brito ESV, Braga JPR. Elementos que influenciam o acesso à atenção primária na perspectiva dos profissionais e dos usuários de uma rede de serviços de saúde do Recife. Physis: Rev Saude Colet 2015; 25(2):635656.
20. Santos CTB, Andrade LOM, Silva MJ, Souza MF. Percurso do idoso em redes de atenção à saúde: um elo a ser construído. Physis 2016; 26(1):45-62.

21. Campos RTO, Ferrer AL, Gama CAP, Campos GWS, Trapé TL, Dantas DV. Avaliação da qualidade do acesso na atenção primária de uma grande cidade brasileira na perspectiva dos usuários. Saúde debate 2014; 38(n. esp.):252-264.

22. Finkler AL, Viera CS, Tacla MTGM, Toso BRGO. O acesso e a dificuldade na resolutividade do cuidado da criança na atenção primária à saúde. Acta Paul Enferm 2014; 27(6):548-553.

23. Magalhães ALA, Morais Neto OL. Desigualdades intraurbanas de taxas de internações por condições sensíveis à atenção primária na região central do Brasil. Cien Saude Colet 2017; 22(6):2049-2062.

24. Gonçalves MR, Hauser L, Prestes IV, Schmidt MI, Duncan BB, Harzheim E. Primary health care quality and hospitalizations for ambulatory care sensitive conditions in the public health system in Porto Alegre, Brazil. Fam Pract 2016; 33(3):238-242.

25. Bastos ML, Menzies D, Hone T, Dehghani K, Trajman A. O impacto da estratégia brasileira de saúde da família sobre condições sensíveis à atenção primária selecionadas: uma revisão sistemática. PLOS One 2017; 12(8): $\mathrm{e} 0182336$.

26. Uscher-Pines L, Pines J, Kellermann A, Gillen E, Mehrotra A. Deciding to visit the emergency department for non-urgent conditions: a systematic review of the Literature. Am J Manag Care 2013; 19(1):47-59.

27. Toso BRGO, Ross C, Sotti CW, Brisch SV, Cardoso JM. Profile of children hospitalizations by primary care sensitive conditions. Acta sci Health Sci 2016; 38(2):231-238.

28. Leão CDA, Caldeira AP, Oliveira MMC. Atributos da atenção primária na assistência à saúde da criança: avaliação dos cuidadores. Rev Bras Saude Matern Infant 2011; 11(3):323-334.

29. Gawryszewski ARB, Oliveira DC, Gomes AMT. Acesso ao SUS: representações e práticas de profissionais desenvolvidas nas Centrais de Regulação. Physis 2012; 22(1):119-140.

Artigo apresentado em 19/01/2020

Aprovado em 01/06/2020

Versão final apresentada em 03/06/2020

Editores-chefes: Romeu Gomes, Antônio Augusto Moura da Silva 